\title{
Confort térmico en vivienda social multifamiliar de clima cálido en Colombia
}

\section{Thermal comfort in multi-family social housing in a warm climate in Colombia}

\section{Walter Giraldo-Castañeda}

Universidad del Valle. Cali (Colombia)

Facultad deArtes Integradas, Escuela de Arquitectura

Departamento de Tecnología de la Construcción

Jorge Daniel Czajkowski

Universidad Nacional de La Plata. La Plata (Argentina)

Facultad de Arquitectura y Urbanismo

Laboratorio de Arquitectura y Hábitat Sustentable

Analía Fernanda Gómez

Universidad Nacional de La Plata. La Plata (Argentina)

Facultad de Arquitectura y Urbanismo

Laboratorio de Arquitectura y Hábitat Sustentable

Giraldo-Castañeda, W., Czajkowski, J. D. y Gómez, A. F. (202I). Confort térmico en vivienda social multifamiliar de clima cálido en Colombia. Revista de Arquitectura (Bogotá), 23(I), II5-I24. https://doi.org/I0.14718/RevArq.202I.2938

\author{
Walter Giraldo-Castañeda \\ Arquitecto, Universidad del Valle. Cali (Colombia) \\ Doctor en Arquitectura y Urbanismo, Universidad Nacional de La Plata. La Plata (Argentina). \\ Magíster en Arquitectura y Urbanismo. Universidad del Valle. Cali (Colombia). \\ Profesor asistente, Universidad del Valle, e investigador con énfasis en arquitectura bio- \\ climática, confort térmico, iluminación natural, ventilación natural y eficiencia energética. \\ https://scholar.google.com/citations?user $=62 \mathrm{LRvHoAAAA} \& \mathrm{hl}=\mathrm{en}$ \\ (D) https://orcid.org/0000-0002-8542-6689 \\ (19)walter.giraldo@correounivalle.edu.co \\ Jorge Daniel Czajkowski \\ Arquitecto, UNLP, La Plata (Argentina) \\ Doctor en Ingeniería, UNLP. La Plata (Argentina). \\ Investigador independiente, CONICET, y director, Laboratorio de Arquitectura y Hábitat \\ Sustentable, UNLP. \\ Profesor Titular UNLP Cátedra Instalaciones I y II, Facultad de Arquitectura y Urbanismo. \\ https://scholar.google.es/citations?user=HN3vinAAAAAJ\&hl=es \\ (D) https://orcid.org/0000-000I-8857-7880 \\ (a) jdczajko@gmail.com \\ Analía Fernanda Gómez \\ Arquitecto, UNLP. La Plata (Argentina). \\ Investigador adjunto, CONICET, y miembro, Laboratorio de Arquitectura y Hábitat Sus- \\ tentable, UNLP. \\ Profesor titular, UNLP, Cátedra Instalaciones I y II, Facultad de Arquitectura y Urbanismo. \\ https://scholar.google.com.ar/citations?user=egw4WHsAAAAJ\&hl=es \\ (D) https://orcid.org/0000-000I-7057-354X \\ (1)anygomez@gmail.com
}

http://dx.doi.org/10.14718/RevArq.2021.2938

\section{Introducción}

\section{Resumen}

El consumo energético residencial en Colombia se incrementó en el $30 \%$ entre 2010 y 2018 , debido, en parte, al gasto destinado a la climatización activa de viviendas de interés social (VIS). Investigaciones sobre VIS unifamiliares reportaron sensaciones térmicas "muy calurosas" con el modelo PMV-PPD en clima cálido. El objetivo de la presente investigación es complementar el estado del arte evaluando el ambiente térmico de una VIS multifamiliar con 2 diferentes modelos de confort: I) el PMV-PPD y 2) el adaptativo. La metodología tuvo 2 fases: I) monitoreo a un ambiente VIS y 2) valoración mediante los dos modelos mencionados. Los resultados indicaron insatisfacción térmica por exceso de calor con ambos modelos en horas diurnas, muy similar a lo reportado en el estado del arte para tipología unifamiliar; sin embargo, también se encontró un ahorro del $35 \%$ en energía dedicada a la climatización si se lo valora con el modelo adaptativo. Se evidenció la ausencia de protección solar en vanos, y los altos valores de temperatura del aire y de la media radiante indicaron que el aislamiento térmico de la envolvente vertical de estas edificaciones es insuficiente para brindar confort térmico. Se proponen mejoras pasivas para mejorar la habitabilidad de estas viviendas y su inclusión obligatoria en el Reglamento 0549 , para reducir el consumo energético y su impacto ambiental.

Palabras clave: Arquitectura bioclimática; construcción sostenible; ASHRAE 55; Modelo PMV-PPD; modelo adaptativo

\begin{abstract}
Residential energy consumption in Colombia increased by $30 \%$ between 2010 and 2018 , due, in part, to spending on active air conditioning of social interest housing (VIS). Research on single-family VIS reported "very hot" thermal sensations with the PMV-PPD model in hot weather. The objective of the present research is to complement the state of the art by evaluating the thermal environment of a multifamily SIV with two different comfort models: I) the PMV-PPD and 2) the adaptive model. The methodology had two phases: I) monitoring of a VIS environment, and 2) assessment using the two models mentioned above. The results indicated thermal dissatisfaction due to excess heat with both models during daytime hours, very similar to what has been reported in the state of the art for single-family typology. However, a $35 \%$ saving in energy dedicated to air conditioning was also found if evaluated with the adaptive model. The absence of solar protection in openings was evidenced, and the high values of air temperature and mean radiant temperature indicated that the thermal insulation of the vertical envelope of these buildings is insufficient to provide thermal comfort. Passive improvements are proposed to ameliorate the habitability of these dwellings and their mandatory inclusion in Regulation 0549, to reduce energy consumption and its environmental impact.
\end{abstract}

Key words: Bioclimatic architecture; sustainable construction; ASHRAE 55; PMV-PPD model; adaptive model

Recibido: septiembre $03 / 2019$
Aceptado: diciembre 16 / 2020
El presente artículo de investigación corresponde a uno de los capítulos iniciales de la tesis doctoral en Arquitectura y Urbanismo de la Universidad Nacional de La Plata, Argentina, titulada: Optimización del confort térmico en clima ecuatorial con tecnologías pasivas en fachadas: el caso de las viviendas de interés social de Cali, realizada por el autor principal bajo la tutoría de los dos coautores, en una comisión de estudio docente otorgada por la Universidad del Valle, de Cali, Colombia. La tesis está vinculada al proyecto de investigación Estrategias de adaptación al cambio climático en edificios y ciudades, y evalúa el confort térmico de las VIS colombianas de clima cálido. Además, la mencionada tesis propondrá y evaluará soluciones constructivas de tipo pasivo, con el fin de mejorar la habitabilidad y, a la par, reducir el consumo energético destinado a climatización. Por su parte, el principal aporte de este artículo es la valoración experimental del confort térmico de las VIS colombianas de tipología multifamiliar en clima cálido, valoración no llevada a cabo aún en el estado del arte.

El fenómeno del calentamiento global requiere ser atendido imprescindiblemente por medio de un cambio paradigmático en la industria de la construcción; dicho cambio debe favorecer la eficiencia energética en las edificaciones para evitar la emisión de gases de efecto invernadero (GEI) generados en la producción de electricidad, ya sea por la quema de combustibles fósiles o por las grandes hidroeléctricas. 
En Colombia, la demanda energética ha tenido un crecimiento cercano al 30\% desde 2010 hasta 2018 (Sistema Intercomunicado Nacional, 2018); la mayor parte de esa demanda está destinada a climatizar activamente las VIS (International Energy Agency, s. f.; Giraldo et al., 2015), con lo que se ha requerido aumentar la infraestructura de grandes hidroeléctricas, las cuales han impactado de manera negativa el medio ambiente (Revista Dinero, 2019).

Las VIS son construidas para garantizar el derecho a la vivienda de los hogares con menores ingresos; además, cumplen con todas las normas urbanísticas (Planeación Municipal, Alcaldía de Santiago de Cali, 2014), arquitectónicas (Ministerio de Ambiente, Vivienda y Desarrollo Territorial, República de Colombia, 2011) y de construcción (MINVIVIENDA, 2000; Ministerio de Minas y Energía, 2009; Asociación Colombiana de Ingeniería Sísmica, 2010; Ministerio de Minas y Energía, 2013), sin sobrepasar un costo para su construcción de 135 salarios mínimos mensuales, equivalentes a USD 36.000 (TRM a 27 de junio de 2018) (Congreso de Colombia, 1997). Según el Departamento Nacional de Estadística (DANE, 2017), las VIS constituyen un porcentaje importante del entorno construido en Colombia, pues equivalen al $49 \%$ de las viviendas; pese a ello, es opcional que este tipo de edificaciones cumpla con el Reglamento de Construcción Sostenible, contenido en la Resolución 0549 de 2015 (Ministerio de Vivienda, Ciudad y Territorio, 2015), la cual aconseja la reducción del consumo energético en el $20 \%$ en relación con una línea base definida por el gobierno.

En el país se distinguen 2 tipologías de VIS: 1) la multifamiliar, o de apartamentos, y 2) la unifamiliar, o de casas. En cantidad, la tipología multifamiliar predomina, con el $68 \%$, sobre la tipología unifamiliar, que representa solo el $32 \%$ (DANE, 2017). En el componente constructivo hay gran variedad de sistemas disponibles (Echeverry Campos y MetroVivienda, 2000); sin embargo el $99 \%$ de ellos están basados en materiales de alta densidad como la mampostería en concreto, la arcilla y los sistemas industrializados, como outinord o contech (García López et al., 2016), que consisten en muros macizos de concreto reforzado con espesores que van desde $8 \mathrm{~cm}$ hasta $12 \mathrm{~cm}$. Dichos sistemas constructivos tienen alta transmitancia térmica, lo cual es negativo para el desempeño térmico de las construcciones en climas cálidos. Esta afirmación se hace respecto a que $10 \mathrm{~cm}$ de muro en concreto equivalen a $4,16 \mathrm{~W} / \mathrm{m}^{2 \circ} \mathrm{K}$, en comparación con los $10 \mathrm{~cm}$ de muro en el sistema de poliestireno expandido de alta densidad, que equivalen a $0,05 \mathrm{~W} / \mathrm{m}^{2} \mathrm{~K}$.
Tomando como base la clasificación climática del Instituto de Hidrología, Meteorología y Estudios Ambientales (IDEAM) incluida en el anexo 2 del Reglamento 0549 de 2015 (Ministerio de Vivienda, Ciudad y Territorio, 2015), más del $80 \%$ del territorio colombiano presenta clima cálido, clasificación en la que se encuentran ciudades tan importantes como Cali, Barranquilla, Cartagena y Buenaventura, entre otras. Para este tipo de clima Olgyay (1998), Givoni (1976) y Santamouris y Asimakopoulos (2001) señalan que se requiere aplicar, principalmente, las estrategias de control solar y ventilación natural para alcanzar el confort térmico; no obstante, cuando estas recomendaciones no se consideran en el diseño arquitectónico ni en la construcción, es casi imposible alcanzar el confort térmico de forma pasiva, como en el caso de las VIS de Cali, Colombia (Giraldo y Herrera, 2017).

De acuerdo con la norma ISO 7730 (International Organization for Standardization, 2005), el confort térmico es el estado mental en el que se expresa satisfacción con el ambiente térmico, y por tal razón las circunstancias requeridas para alcanzarlo no son iguales para todos; sin embargo, mediante investigaciones de laboratorio y de campo se han podido establecer estadísticamente las condiciones en las que la mayoría de las personas son térmicamente confortables.

Las principales variables que influyen en el confort térmico son: la temperatura del aire ( $\left.\mathrm{T}_{\text {air }}\right)$, la humedad relativa $(\mathrm{Hr})$, la velocidad del aire $\left(\mathrm{V}_{\text {air }}\right)$, la temperatura media radiante $(\mathrm{Tmr})$, el nivel de vestimenta (Clo) y la tasa metabólica (Met). Pioneros en el tema, como Olgyay (1998), Givoni (1976) y Fanger (1970), propusieron modelos para valorar el confort con dichas variables.

El modelo de Fanger es muy utilizado por incorporar todas las variables ya mencionadas, en dos índices: 1) el Predicted Mean Vote (PMV), que indica la sensación térmica en una escala de 7 valores que va desde -3, o "muy frío", a +3, o "muy caluroso", y 2) el Predicted Percentage Dissatisfied (PPD), que señala el porcentaje de personas insatisfechas en el ambiente. En este modelo, la situación ideal de confort térmico es un valor PMV de 0, o "Neutro", y un PPD no mayor que el $20 \%$. Este modelo ha sido adoptado en estándares internacionales como: ISO 7730, desde 1984, y el ASHRAE Standard 55, desde 1992 (American Society of Heating Refrigerating and Air Conditioned Engineers [ASHRAE], 2017). También ha sido adoptado en el ámbito nacional en el Reglamento 0549 de Construcción Sostenible (Ministerio de Vivienda, Ciudad y Territorio, 2015). Es ampliamente 
aceptado para evaluar ambientes con climatización mecánica, porque numerosas investigaciones han encontrado discrepancias; sobre todo, en edificios ventilados naturalmente, en los cuales los ocupantes expresan satisfacción térmica en un rango más amplio (Carlucci et al., 2018; Forgiarini Rup et al., 2015). Por lo expuesto, el modelo adaptativo fue incluido en versiones posteriores de las mismas normas.

En particular, el modelo de Fanger establece un rango de confort estático para la Tair entre 21 ${ }^{\circ} \mathrm{C}$ y $25{ }^{\circ} \mathrm{C}$, sin considerar el clima evaluado; en contraste el modelo adaptativo considera confortables, incluso, $3,5{ }^{\circ} \mathrm{C}$ por encima y por debajo de la $T_{a i r}$ media del clima evaluado.

El modelo adaptativo tiene como parámetro de evaluación la temperatura operativa ( $\left.T_{\mathrm{op}}\right)$, que es una ponderación entre la $T_{a i r}$ y la $T_{m r}$. Por ejemplo, para un clima con $25{ }^{\circ} \mathrm{C}$ de $T_{\text {air }}$ media, como Cali, la situación ideal es que su $\mathrm{T}_{\mathrm{op}}$ se encuentre en ese mismo valor; sin embargo, están dentro del rango de aceptabilidad valores entre $29^{\circ} \mathrm{C}$ y $22{ }^{\circ} \mathrm{C}$.

Brindar confort térmico de forma pasiva en las viviendas tiene una ventaja innata, y es la del bienestar y la salubridad (Ormandy y Ezratty, 2012; Kaushik et al., 2020); sin embargo, hay otras, como la económica y la sostenible. La ventaja económica está dada por los costos ahorrados en consumo energético, que son muy bien recibidos, especialmente, por la comunidad de viviendas sociales; mientras, la sostenible se da por la disminución del calentamiento global, pues al reducirse el consumo energético también se reducen las emisiones de GEI, pues se estima que la mitad de la energía producida en el mundo es consumida por los hogares, y de esa mitad, cerca del $40 \%$ se dedica a dispositivos activos de climatización (United Nations Commission on Human Settlements, ONU, 2011).

Gamboa et al. (2011) evaluaron el confort térmico en VIS de Cali. En el ámbito urbano se concluyó que este podría mejorarse potenciando microclimas que reduzcan el fenómeno de la isla de calor urbana mediante paisajismo. En el ámbito arquitectónico, por medio de encuestas y monitoreos, determinaron que la insatisfacción térmica es generalizada en la tipología unifamiliar, en la cual registraron sensaciones térmicas "muy calurosas" y un PPD que llegó hasta el 100\%. Mediante termografías, revelaron altos niveles de temperaturas superficiales en las envolventes, como cubiertas y fachadas, que alcanzan hasta $49{ }^{\circ} \mathrm{C}$. Finalmente, los autores demostraron, con la aplicación de una barrera radiante instalada bajo la cubierta, que es posible alcanzar el confort de manera económica en esta tipología.

Iturre (2013) trabajó con las VIS de Buenaventura, de clima cálido húmedo. Su trabajo identificó, mediante monitoreos y encuestas en más de 200 viviendas, que en el $53 \%$ de ellas se utilizaba constantemente climatización mecánica para lograr confort durante el día y parte de la noche. Según el autor, esto se debe, principalmente, al calor ganado por la cubierta y por su baja resistencia térmica. Además, el autor encontró ausencia de ventilación cruzada, alta absortividad y baja resistencia térmica en los muros de fachada. La solución teórica propuesta para mejorar tal condición consistió en mejorar la resistencia térmica de la cubierta, cambiar el color de los muros de fachada para reducir la absortividad y generar ventilación cruzada creando nuevos vanos.

Igualmente, Barona (2016) determinó el comportamiento térmico de los dos sistemas constructivos de fachada más utilizados en las VIS de Cali: mampostería estructural con bloques de arcilla cocida y muros macizos de concreto reforzado. Dicho estudio se llevó a cabo en viviendas unifamiliares construidas en municipios aledaños a Cali, como Candelaria y Jamundí, los cuales comparten las mismas características climáticas. Mediante monitoreo y cálculos matemáticos, Barona determinó las condiciones térmicas de las viviendas y evaluó el confort térmico mediante el índice de Fanger. Se concluyó que las sendas envolventes verticales no poseen la capacidad suficiente para aislar térmicamente el calor irradiado por el sol y brindar confort térmico, ya que la sensación térmica más común fue la "muy calurosa", lo cual significa un porcentaje de insatisfechos superior al $90 \%$.

Por último, Giraldo et al. (2015) llevaron a cabo un análisis de simulación computacional con un modelo multifamiliar VIS con una envolvente de concreto reforzado de 0,08 $\mathrm{m}$ de espesor. Los autores identificaron que desde las 10:00 hasta las 21:00 la sensación térmica presentada en un apartamento se encuentra fuera de los rangos de confort. Proponen también algunas soluciones, que consisten en el aumento de la resistencia térmica de la envolvente, y protección solar a las ventanas. Estas mejoras significaron una reducción del $60 \%$ de la carga térmica en el interior del apartamento; además, representaron en la construcción inicial tan solo un sobrecosto del $16 \%$, el cual se puede recuperar en 3 años de funcionamiento.

Los trabajos experimentales de Gamboa et al. (2011), Iturre (2013) y Barona (2016), realizados todos en clima cálido, coinciden en señalar insatisfacción térmica en las VIS unifamiliares construidas con los sistemas de mampostería de ladrillo o concreto, lo cual permite generalizar este desempeño. En contraste, para la tipología multifamiliar, tan solo se cuenta con el estudio computacional hecho por Giraldo et al. (2015); por lo tanto, es necesario continuar el análisis térmico de la tipología VIS multifamiliar, entre otras cosas, porque supera ampliamente en cantidad a la unifamiliar en todo el país, y su ganancia térmica es muy diferente. En la tipología unifamiliar la carga térmica recibida por la cubierta es notablemente mayor, debido a que esta constituye la mayor área de la envolvente; mientras, en la tipología multifamiliar el calor ganado por la cubierta afecta únicamente a las unidades habitacionales del nivel más alto. En los bloques de múltiples apartamentos la mayor área de la envolvente corresponde a las fachadas, las cuales abarcan la totalidad de los apartamentos. 
Así mismo, estos trabajos han evaluado el confort térmico mediante el modelo PMV-PPD, a pesar de que dichas viviendas son ventiladas naturalmente. El presente trabajo propone evaluar el confort térmico con ambos modelos: el PMV-PPD, para tener la misma escala valorativa de los estudios anteriores, y también el adaptativo, para tener valoraciones más precisas y confrontar resultados desde el punto de vista de la eficiencia energética y la sostenibilidad.

Por lo tanto, el objetivo principal planteado en este trabajo es evaluar el nivel de confort térmico brindado por un ambiente de la tipología multifamiliar de la VIS de Santiago de Cali, Colombia, donde se presenta un clima cálido seco, mediante un monitoreo de campo y uso comparativo de los modelos PMV-PPD y adaptativo.

\section{Metodología}

\section{Edificación experimental}

El edificio escogido para el presente estudio es un bloque de cinco niveles de apartamentos perteneciente al conjunto residencial Calicanto, localizado al sur de la ciudad de Santiago de Cali, entre las calles 42 y 45 y las carreras 95 A y 96 A. El sistema constructivo de dicha edificación es de mampostería reforzada de bloques estructurales de arcilla cocida a la vista con revoque y pintura en el interior; tiene, además, una relación de ventana pared del $30 \%$ y un aspecto en planta 1:1,7. El ambiente monitoreado corresponde a la alcoba principal de un apartamento de $56 \mathrm{~m}^{2}$ ubicado en la esquina suroeste del cuarto nivel de la edificación

La figura 1 contiene a la izquierda la planta arquitectónica del cuarto nivel, donde se señala, mediante sombreado, la ubicación del apartamento en el edificio. Mientras, a la derecha se muestra la distribución arquitectónica del apartamento, en la cual se señala, también con un sombreado, el dormitorio de interés, el cual tiene dimensiones intramuros de 2,7 $\mathrm{m} \times 3,0 \mathrm{~m}$. En la figura 2 se muestra una vista exterior del bloque de apartamentos; se indica, con línea discontinua de color negro, la unidad de vivienda analizada.

experimental. Plantas arquitectónicas: cuarto nive del bloque; se resalta el apartamento seleccionado (izquierda) y del apartamento señalando el ambiente monitoreado (derecha). Fuente: elaboración propia (2019).

\section{Obtención de datos}

En la obtención de datos se procuró seguir la norma ISO 7726 (International Organization for Standardization, 2002). Las variables ambienta- les, como $T_{a i r}, H r$, $V_{\text {air }}$ y $T_{m r}$, fueron adquiridas mediante sensores, mientras que para las variables personales, como Clo y Met, se determinó un perfil dinámico de acuerdo con las costumbres de los ocupantes.

Para obtener la Tair y la Hr, un termohigrómetro digital con almacenamiento de datos (datalogger) registró cada hora estas variables en frente de la cama, a una altura de 1,1 m. Este sensor fue protegido de la radiación mediante una cajiIla perforada de aluminio pulido.

Los datos de $V_{\text {air }}$ fueron obtenidos mediante un anemómetro de hilo caliente posicionado en el área operable de la ventana corrediza de doble panel. Con este equipo se tomó constantemente la velocidad del aire y se elaboró un promedio de estos registros cada hora. Durante la medición, la ventana fue operada libremente a voluntad de los ocupantes, pero la puerta permaneció cerrada.

Por su parte, la $\mathrm{T}_{\mathrm{mr}}$ se obtuvo registrando cada hora las temperaturas superficiales de los seis planos que conforman la habitación, con un termómetro infrarrojo, para posteriormente ser calculada mediante la siguiente fórmula, propuesta por Dunkle (1963), y posteriormente seguida por ASHRAE (American Society of Heating Refrigerating):

$$
T_{m r}=\left[\sqrt[4]{\sum_{n} f p \varepsilon(t i+273,15)^{4}}\right]-273,15
$$

\section{Donde:}

$t i=$ temperatura de la superficie $i$ en ${ }^{\circ} \mathrm{C}$.

$f p=$ factor de vista entre el punto central de la habitación y la superficie

$\varepsilon=$ emisividad del material.

La figura 3 muestra una vista interior del dormitorio, con un esquema de la instalación de los sensores, y en la tabla 1 se relaciona cada variable con el instrumento utilizado para su medición y sus especificaciones.

Por último, la tasa metabólica (Met) y el nivel de vestimenta $(\mathrm{Clo})$ se determinaron mediante la observación de los hábitos cotidianos de los
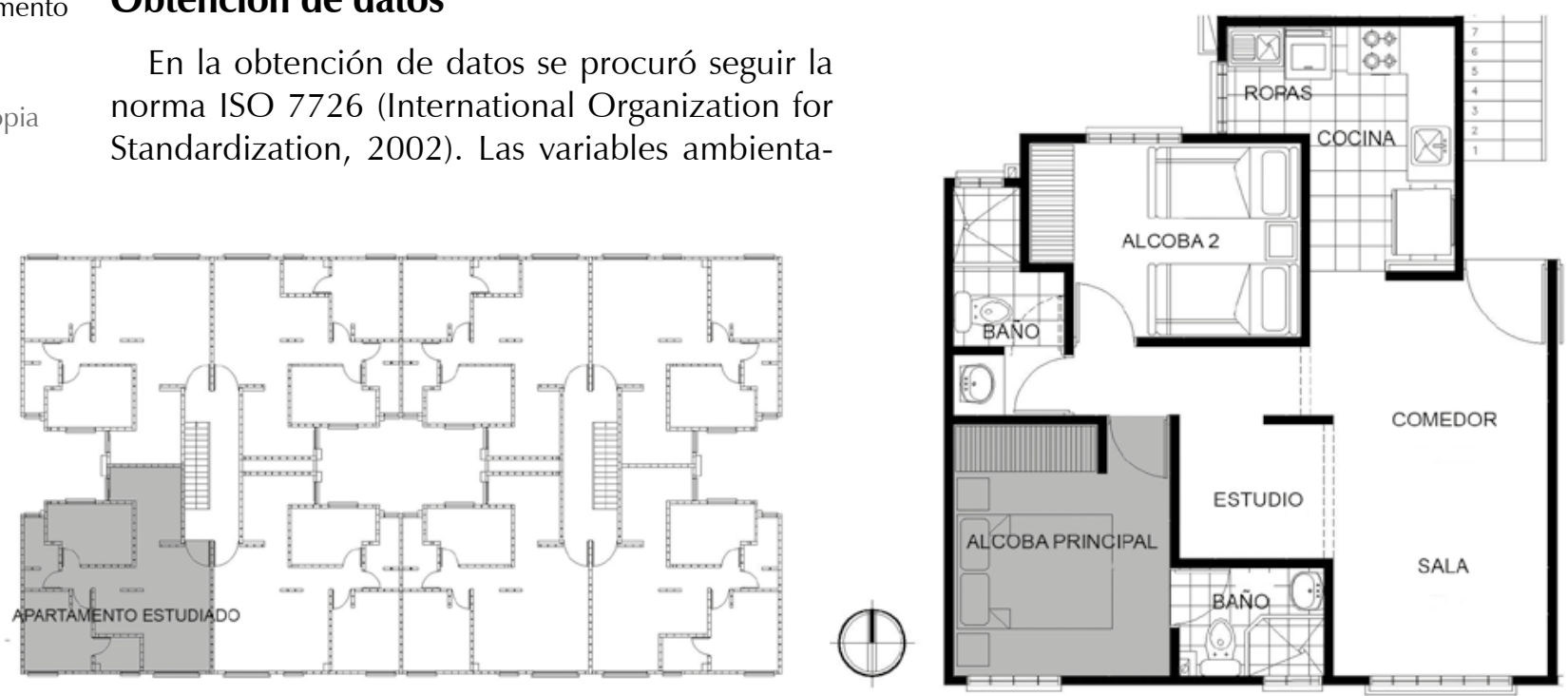
ocupantes y la asignación posterior de valores de acuerdo con el ASHRAE Handbook Fundamentals (ASHRAE, 2001). La valoración se dividió en dos etapas: 1) la de actividades y 2) la de descanso.

\section{Periodo de monitoreo}

El monitoreo se llevó a cabo durante la primera semana de enero de 2016. De acuerdo con las bases de datos climáticas (Instituto de Hidrología, Meteorología y Estudios Ambientales, Tiempo y Clima en Colombia [IDEAM], 2016), este es uno de los periodos donde se reúnen altos valores de radiación solar y temperatura del aire. Adicionalmente, en esta época del año el dormitorio analizado no solo recibe radiación solar directa por la fachada oeste, sino que también la recibe por la fachada sur, lo que configura un periodo crítico de carga térmica externa.

\section{Procesamiento de datos}

Con los datos obtenidos durante la semana se elaboró un día típico conformado por los promedios horarios de cada variable. Posteriormente, los valores de este día típico fueron introducidos al software PMV Calc v2 (Department of Technology and Built Environment - Laboratory of Ventilation and Air Quality - University of Gävle, s. f.), de uso libre, para calcular hora a hora los índices PMV y PPD.

Por otro lado, para evaluar el confort térmico mediante el modelo adaptativo se preparó un gráfico basado en la figura 5.3 del ASHRAE Standard 55, que relaciona en el eje vertical la $\mathrm{T}_{\mathrm{op}}$ y en el eje horizontal la Tair media (ASHRAE, 2017); esta gráfica, a su vez, depende de una hoja electrónica que calcula la temperatura operativa con los valores de $\mathrm{T}_{\text {air }}$ y $\mathrm{T}_{\mathrm{mr}}$ del día típico mediante la siguiente fórmula:

$$
\text { Top }=A^{*} t a+(1-A) * t m r
$$

Donde:

$$
\begin{aligned}
& T_{\mathrm{op}}=\text { Temperatura operativa } \\
& A=\text { Coeficiente de velocidad del aire } \\
& t \mathrm{a}=\text { Temperatura del aire } \\
& t m r=\text { Temperatura media radiante }
\end{aligned}
$$

Posteriormente, la $\mathrm{T}_{\mathrm{op}}$ se une con la temperatura exterior media predominante de Cali $\left(25^{\circ} \mathrm{C}\right)$, la cual fue calculada con la base de datos de un año de mediciones de la estación meteorológica de la Escuela de Arquitectura de la Universidad del Valle de Cali, en su sede de Meléndez.

\section{Resultados}

\section{Temperatura del aire}

La figura 4 muestra el comportamiento de la $T_{\text {air }}$ durante la semana de medición; en esta se señala el rango de confort, de entre $22{ }^{\circ} \mathrm{C}$ y $29,5{ }^{\circ} \mathrm{C}$, recomendado por Olgyay (1998) y Givoni (1976), mediante el área sombreada. La $\mathrm{T}_{\text {air }}$ máxima registrada fue de $34,7{ }^{\circ} \mathrm{C}$, y la mínima, de $20,9^{\circ} \mathrm{C}$. El límite superior del rango de confort fue superado notoriamente en 5 de los 7 días monitoreados, por lo general, desde las 10:00 hasta las 18:00. Nótese cómo dicho periodo coincide con las horas del día en las cuales se recibe radiación solar en la fachada sur y oeste, con lo que se infiere el déficit de estrategias de protección solar para muros y

\begin{tabular}{|c|c|}
\hline Variable & Instrumento \\
\hline $\begin{array}{l}\text { Temperatura del } \\
\quad \text { aire. } \\
\text { Humedad relativa. }\end{array}$ & $\begin{array}{l}\text { Termohigrómetro digital (datalogger) marca Extech (modelo } \\
\text { RHT30). Sensibilidad a un rango de } \mathrm{T}_{\text {air }} \text { entre }-30^{\circ} \mathrm{C} \text { y } 70{ }^{\circ} \mathrm{C} \text {, } \\
\text { con una resolución de } 0,1^{\circ} \mathrm{C} \text { y precisión de } \pm 0,5^{\circ} \mathrm{C} \text {. En } \\
\text { Cuanto a la } \mathrm{Hr} \text {, el equipo tiene un rango de medición entre } \\
0,1 \% \text { y } 99,9 \% \text {, con resolución de } 0,1 \% \text { y precisión de } \pm 3 \% \text {. }\end{array}$ \\
\hline $\begin{array}{l}\text { Temperatura } \\
\text { superficial o } \\
\text { temperatura } \\
\text { radiante }(\mathrm{Tr}) .\end{array}$ & $\begin{array}{l}\text { Termómetro infrarrojo marca Extech modelo IR } 42500 \text {. El rango } \\
\text { de medición va desde }-20{ }^{\circ} \mathrm{C} \text { a } 260{ }^{\circ} \mathrm{C}, \\
\text { con una precisión del } 98 \% \text { al } 97 \%\end{array}$ \\
\hline Velocidad del aire. & $\begin{array}{c}\text { Termoanemómetro digital de hilo caliente marca CEM (modelo } \\
\text { DT-8880). Rango de medición desde 0,01 hasta } 25 \mathrm{~m} / \mathrm{s} \text {. } \\
\text { Resolución de } 0,01 \mathrm{~m} / \mathrm{s} \text {. }\end{array}$ \\
\hline
\end{tabular}

(1) Tabla 1. Especificación del instrumental utilizado.

Fuente: elaboración propia (2019).
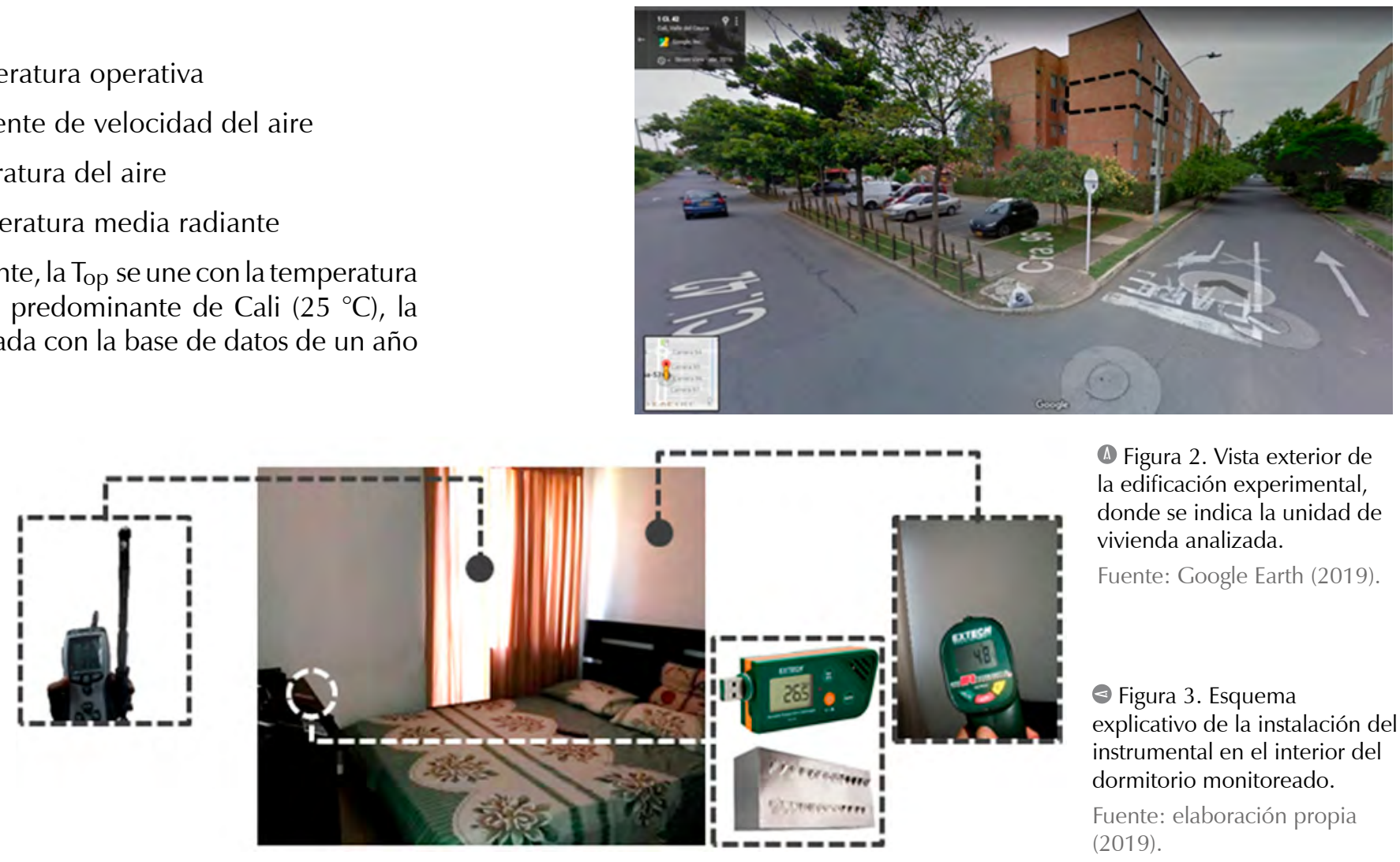

(4) Figura 2. Vista exterior de la edificación experimental, donde se indica la unidad de vivienda analizada. Fuente: Google Earth (2019).

- Figura 3. Esquema explicativo de la instalación del instrumental en el interior del dormitorio monitoreado. Fuente: elaboración propia (2019). 
ventanas. Además, la Tair del dormitorio estuvo levemente por debajo del rango de confort en 3 de los 7 días monitoreados; generalmente, desde las 4:00 hasta las 6:00. Las condiciones de la Tair durante este periodo son ideales para enfriar los espacios mediante ventilación natural nocturna, con el objeto de que estén más frescos a la hora de recibir las cargas térmicas de la mañana y la tarde.

\section{Humedad relativa}

La figura 5 muestra el comportamiento de la $\mathrm{H}_{\mathrm{r}}$ durante los 7 días de monitoreo. En esta se señala el rango de confort propuesto por Olgyay (1998), de entre el $30 \%$ y el $70 \%$, mediante la zona sombreada. La Hr máxima alcanzada fue del $84 \%$, y la mínima, del $34 \%$. El espacio presenta exceso de humedad en 6 de los 7 días monitoreados en las horas de la madrugada. Este superávit de humedad es poco problemático, debido a que, generalmente, este es el periodo de descanso y se encuentra acompañado por Tair confortables; por lo tanto, evaporar sudoración en exceso no es necesario.

\section{Velocidad del aire}

La figura 6 muestra el comportamiento de la

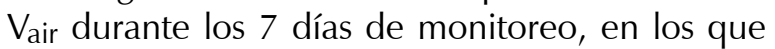

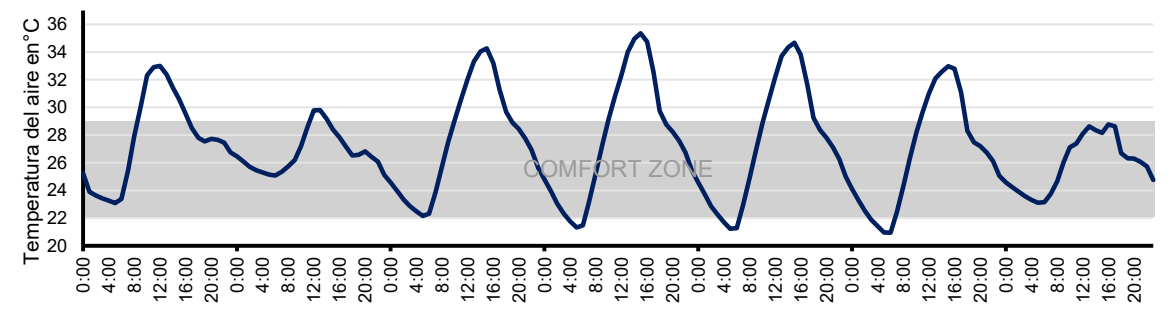

(4) Figura 4. Temperatura del aire durante los siete días de monitoreo.

Fuente: elaboración propia (2019).

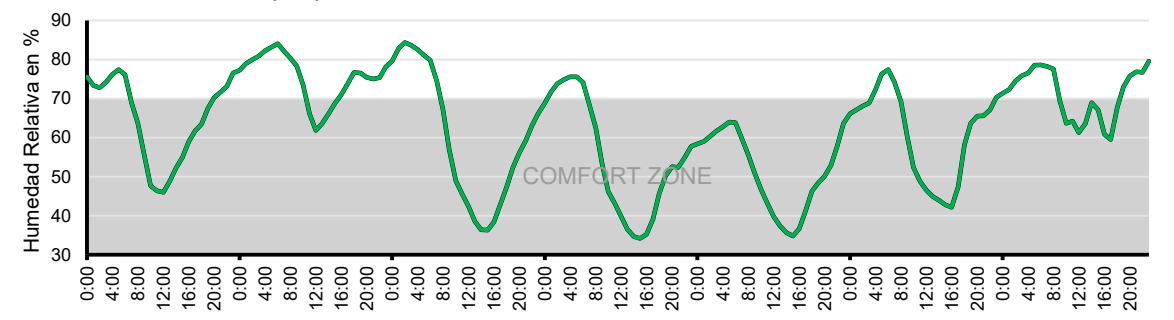

(4) Figura 5. Humedad relativa durante los siete días de monitoreo.

Fuente: elaboración propia (2019).

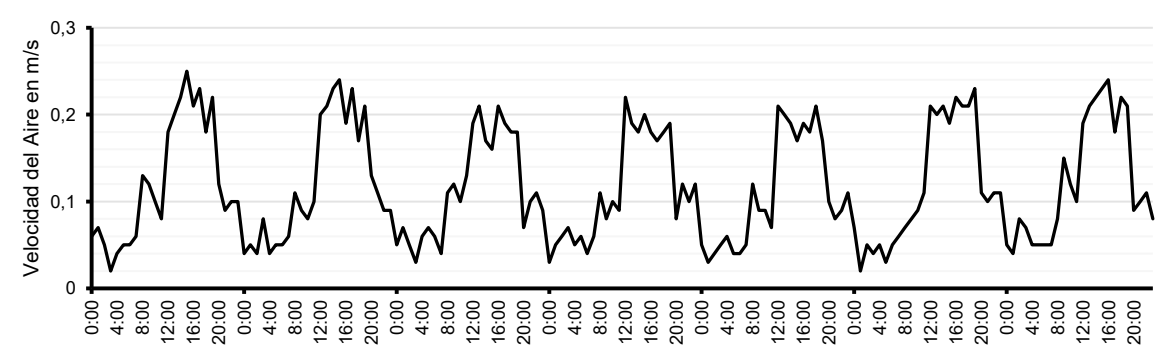

(4) Figura 6. Velocidad del aire durante los siete días de monitoreo.

Fuente: elaboración propia (2019).

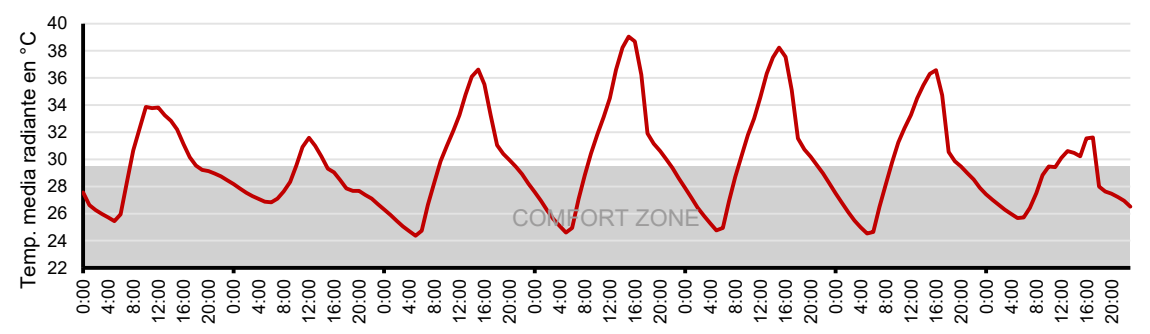

(4) Figura 7. Temperatura media radiante durante los siete días de monitoreo.

Fuente: elaboración propia (2019). la ventana fue manipulada libremente a elección de los ocupantes (generalmente permaneció por completo abierta en las horas de la mañana y la tarde, y parcialmente, durante la noche y la madrugada), pero la puerta permaneció cerrada. La gráfica muestra que la $V_{\text {air }}$ en el espacio fue menor durante las horas de la madrugada, y se incrementó durante las horas del mediodía y la tarde. Pese a ello, los registros oscilaron entre 0,02 $\mathrm{m} / \mathrm{s}$ y $0,25 \mathrm{~m} / \mathrm{s}$, lo cual es calificado por Olgyay (1998) como viento en calma o inadvertido.

Estos bajos valores de velocidad del aire se atribuyen a que la habitación no cuenta con ventilación cruzada, pues tiene tan solo una ventana corrediza, en la cual solo es útil el $50 \%$ de su área, por la cual el aire debe entrar y salir a la vez. Lamentablemente, esta condición de tipo de ventilación y tipo de ventana es característica general de las VIS.

\section{Temperatura media radiante}

La figura 7 muestra el comportamiento de la $\mathrm{T}_{\mathrm{mr}}$ durante los siete días de monitoreo. En esta se señala el rango de confort propuesto por Olgyay (1998) y Givoni (1976), de entre $22^{\circ} \mathrm{C}$ y 29,5 ${ }^{\circ} \mathrm{C}$, mediante la franja sombreada. La $\mathrm{T}_{\mathrm{mr}}$ máxima alcanzada fue de $38,7^{\circ} \mathrm{C}$, y la mínima, de 24,36 ${ }^{\circ} \mathrm{C}$. El límite superior del rango de confort se superó en todos los días monitoreados, desde las horas de la mañana hasta el final de la tarde o, en algunos casos, hasta la noche.

Cabe resaltar que la figura 7 representa la $\mathrm{T}_{\mathrm{mr}}$ en la cual se computan las temperaturas superficiales de todo el dormitorio (muros, piso y techo) con la ecuación ya indicada; sin embargo, la temperatura superficial de la fachada oeste fue la que alcanzó el valor más alto, de hasta $48{ }^{\circ} \mathrm{C}$, en el cuarto día de medición.

Al integrar lo expuesto en la figura 1 con la proximidad de la cama a la fachada oeste y sus $48{ }^{\circ} \mathrm{C}$ de temperatura superficial, es claro que el cuerpo de los usuarios es irradiado muy directamente por su calor acumulado. Esta deficiente condición para el confort térmico es ocasionada por la disposición del mobiliario planteada en el diseño arquitectónico (nótese que es la única posición adecuada para la cama), las pequeñas dimensiones en planta de la alcoba y las altas absortividades y transmitancias térmicas de la envolvente. Infortunadamente, al menos los últimos 3 factores son características generales de las VIS colombianas.

\section{Nivel de vestimenta}

De acuerdo con lo observado durante el monitoreo y los valores de Clo consignados en el Standard ASHRAE Handbook Fundamentals (tabla 8, capítulo 8) (ASHRAE, 2001), para las horas comprendidas entre las 8:00 y las 21:00 los usuarios usaron pantalones ligeros, camisa de manga corta y calzado liviano, lo cual equivale a un Clo de 0,6 . Mientras, para las horas de descanso, de las 22:00 a las 7:00 utilizaron piyama y frazada, lo cual equivale a un valor de 1,2 Clo. 
Nótese que los niveles de vestimenta son dinámicos y se adaptan a las condiciones ambientales que, en definitiva, son percibidas por los usuarios. En este caso, el uso de mayor cantidad de aislamiento térmico en la vestimenta utilizada en las horas de la noche se atribuye, principalmente, a la reducción de la $T_{\text {air }} y$ de la $T_{\mathrm{mr}}$.

\section{Tasa metabólica}

De acuerdo con las actividades llevadas a cabo por los usuarios y con los valores Met consignados en el standard ASHRAE Handbook Fundamentals (tabla 4, capítulo 8) (ASHRAE, 2001), los usuarios desarrollaron actividades físicas moderadas desde las 8:00 hasta las 21:00, lo cual equivale a un valor de 1,2; mientras, el tiempo de las 22:00 a las 8:00 fue dedicado a dormir, lo cual equivale a un valor de 0,8 .

\section{Evaluación de confort con el modelo de Fanger PMV-PPD}

Con base en los promedios de $\mathrm{T}_{\mathrm{air}}, \mathrm{H}_{\mathrm{r}}, \mathrm{V}_{\text {air }} \mathrm{y}$ $T_{m r}$ obtenidos en los siete días de medición, y con los valores de Clo y Met determinados, se elaboró un día típico, con el cual posteriormente se valoró el confort térmico mediante los índices PMV y PPD.

La tabla 2 contiene los valores medios de cada variable en cada una de las 24 horas del día típico con los que se calcularon dichos índices. El PMV y PPD resultantes en cada hora se encuentran en las dos columnas finales.

Finalmente, para revisar de manera visual estos datos, se elaboraron las figuras 8 y 9 . La figura 8 muestra el comportamiento del PMV; allí se distingue que el espacio logra únicamente sensaciones térmicas cercanas a lo "neutro" desde las 23:00 hasta las 8:00; mientras, desde las 9:00 hasta las 22:00 se sobrepasa el límite de lo "ligeramente caluroso", y permanece, por tanto, entre las sensaciones "calurosas" y "muy calurosas". Nótese también que entre las 13:00 y las 17:00 se alcanza la sensación "muy calurosa", que equivale al nivel más alto de insatisfacción por exceso de calor.

Esta información se complementa con el porcentaje de personas insatisfechas o PPD, que se muestra en la figura 9; en esta se nota que en 14 de las 24 horas del día el espacio permaneció por fuera del rango "aceptable" de confort térmico, establecido en el 20\%, y llegó a cifras de insatisfechos de hasta el 93,4\%, entre las 13:00 y las 17:00.

\section{Evaluación de confort con el modelo adaptativo}

La figura 10 muestra la evaluación de confort mediante el modelo adaptativo. Esta se elaboró calculando las temperaturas operativas horarias con base en la ponderación entre $T_{a i r}, y_{m r}$ del día típico y teniendo en cuenta que la temperatura exterior media predominante de Cali es de $25{ }^{\circ} \mathrm{C}$.

De la figura 10 se infiere que, dentro de los límites de aceptabilidad, del $80 \%$, se encuentra el periodo comprendido entre las 19:00 y las 9:00, lo cual equivale a 15 horas de satisfacción; mientras, por fuera de este mismo límite se encuentra el periodo comprendido entre las 10:00 y las 18:00, lo cual equivale a 9 horas de insatisfacción. En este periodo de insatisfacción se alcanzó una separación máxima de hasta $4{ }^{\circ} \mathrm{C}$ de los límites de aceptabilidad de temperatura operativa entre las 14:00 y las 15:00.

Cabe destacar que la cantidad de tiempo por fuera del límite de aceptabilidad del $80 \%$ difiere en 5 horas de un modelo evaluativo a otro; es decir, con el modelo PMV-PPD se tienen 14 horas por fuera del rango, mientras que con el modelo adaptativo solo se obtienen 9, con lo cual el tiempo de funcionamiento de los equipos de climatización activa se reduce en el $35 \%$ con este último modelo, porque tiene rangos de confort más amplios.

\section{Discusión}

Como bien se indicó en la Introducción, Olgyay (1998), Givoni (1976) y Santamouris y Asimakopoulos (2001) dan prioridad a las estrategias bioclimáticas de control solar y ventilación natural en los climas cálidos. En cuanto a la primera de esas dos estrategias, como lo mostró la figura 2, el edificio tipo VIS objeto de observación carece de elementos de protección solar en vanos como aleros o quiebrasoles, y contrariamente a las recomendaciones de usar colores claros para reducir la absorción de radiación solar, sus muros exteriormente dejan a la vista el oscuro bloque de arcilla. De la misma forma, hace parte de esta estrategia la transmitancia térmica de los muros, la cual, de acuerdo con sus componentes, alcanza $2,37 \mathrm{~W} / \mathrm{m}^{2} \mathrm{~K}$, mientras que el Reglamento 0549 de Construcción Sostenible (Ministerio de Vivienda, Ciudad y Territorio, 2015) parte de la base de tener, como máximo, $1,21 \mathrm{~W} / \mathrm{m}^{2 \circ} \mathrm{K}$.

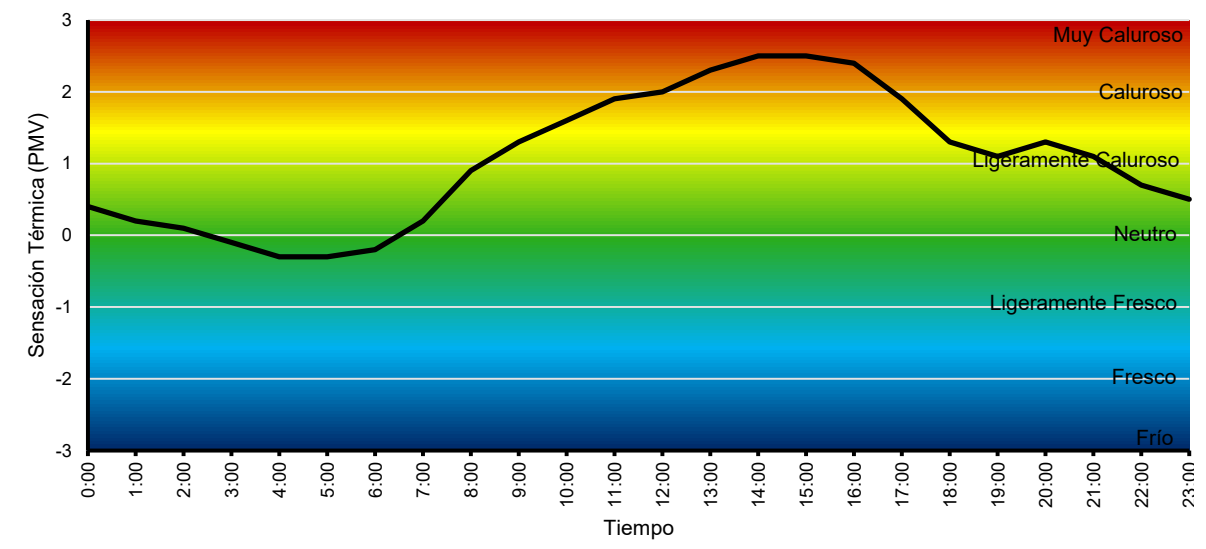

(4) Figura 8. Gráfica de PMV (índice de sensación térmica) en el día típico. Fuente: elaboración propia.

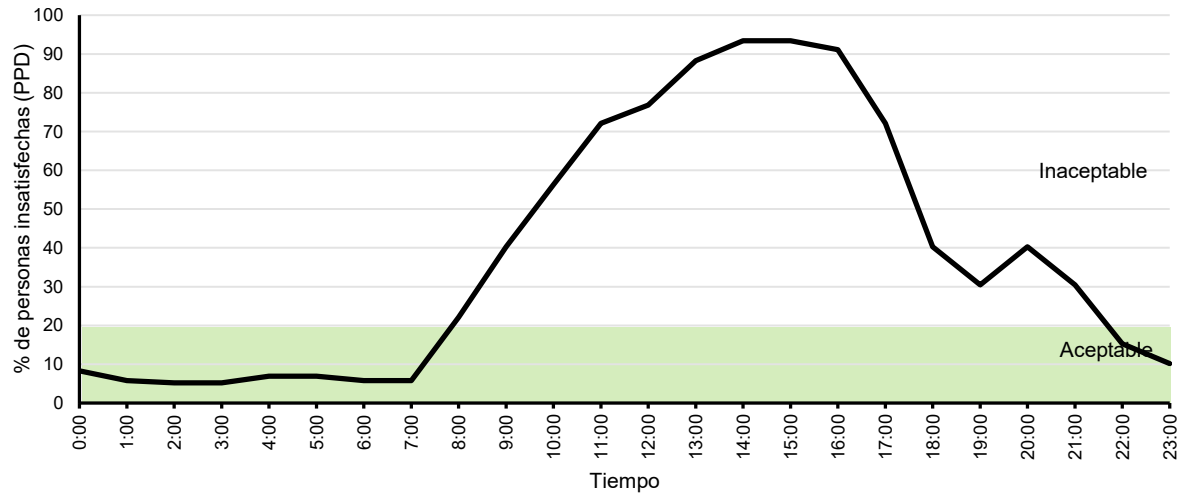

(4) Figura 9. Gráfica de PPD (Porcentaje de personas insatisfechas) en el día típico. Fuente: elaboración propia (2019). 
๑ Tabla 2. Día típico elaborado con los valores medios horarios de cada variable y los valores de PMV y PPD obtenidos.

Fuente: elaboración propia.

(1) Figura 10. Gráfica de evaluación de confort térmico mediante el modelo adaptativo en el día típico.

Fuente: elaboración propia

\begin{tabular}{|c|c|c|c|c|c|c|c|c|c|}
\hline Fecha & Hora & $\mathrm{T}_{\mathrm{air}}$ & $\mathrm{T}_{\mathrm{mr}}$ & $V_{\text {air }}$ & HR & Met & Clo & PMV & PPD \\
\hline Ene 02 -Ene 08 & 0:00:00 & 24,7 & 27,0 & 0,05 & 71,01 & 0,8 & 1,2 & 0,40 & 8,30 \\
\hline Ene 02 -Ene 08 & 1:00:00 & 23,9 & 26,6 & 0,05 & 72,18 & 0,8 & 1,2 & 0,2 & 5,80 \\
\hline Ene 02 -Ene 08 & 2:00:00 & 23,5 & 25,9 & 0,05 & 73,40 & 0,8 & 1,2 & 0,1 & 5,20 \\
\hline Ene 02 -Ene 08 & 3:00:00 & 23,0 & 25,5 & 0,05 & 74,25 & 0,8 & 1,2 & $-0,10$ & 5,20 \\
\hline Ene 02 -Ene 08 & 4:00:00 & 22,3 & 24,9 & 0,05 & 75,44 & 0,8 & 1,2 & $-0,30$ & 6,90 \\
\hline Ene 02 -Ene 08 & 5:00:00 & 22,4 & 24,6 & 0,05 & 76,57 & 0,8 & 1,2 & $-0,30$ & 6,90 \\
\hline Ene 02 -Ene 08 & $6: 00: 00$ & 22,5 & 25,2 & 0,05 & 76,28 & 0,8 & 1,2 & $-0,20$ & 5,80 \\
\hline Ene 02 -Ene 08 & 7:00:00 & 23,7 & 26,9 & 0,05 & 72,30 & 0,8 & 1,2 & 0,20 & 5,80 \\
\hline Ene 02 -Ene 08 & 8:00:00 & 25,2 & 28,5 & 0,10 & 68,00 & 1,2 & 0,6 & 0,90 & 22,10 \\
\hline Ene 02 -Ene 08 & 9:00:00 & 27,15 & 29,7 & 0,10 & 60,66 & 1,2 & 0,6 & 1,30 & 40,30 \\
\hline Ene 02 -Ene 08 & 10:00:00 & 28,5 & 30,7 & 0,10 & 54,12 & 1,2 & 0,6 & 1,60 & 56,30 \\
\hline Ene 02 -Ene 08 & 11:00:00 & 29,7 & 31,7 & 0,10 & 51,03 & 1,2 & 0,6 & 1,90 & 72,10 \\
\hline Ene 02 -Ene 08 & 12:00:00 & 30,7 & 32,4 & 0,20 & 48,20 & 1,2 & 0,6 & 2,00 & 76,80 \\
\hline Ene 02 -Ene 08 & 13:00:00 & 31,5 & 33,4 & 0,20 & 47,60 & 1,2 & 0,6 & 2,30 & 88,30 \\
\hline Ene 02 -Ene 08 & 14:00:00 & 32,0 & 34,4 & 0,20 & 48,25 & 1,2 & 0,6 & 2,50 & 93,40 \\
\hline Ene 02 -Ene 08 & 15:00:00 & 31,8 & 34,6 & 0,20 & 48,40 & 1,2 & 0,6 & 2,50 & 93,40 \\
\hline Ene 02 -Ene 08 & 16:00:00 & 31,3 & 34,2 & 0,20 & 49,00 & 1,2 & 0,6 & 2,40 & 91,10 \\
\hline Ene 02 -Ene 08 & 17:00:00 & 29,8 & 32,7 & 0,20 & 52,20 & 1,2 & 0,6 & 1,90 & 72,10 \\
\hline Ene 02 -Ene 08 & 18:00:00 & 28,3 & 30,0 & 0,20 & 57,90 & 1,2 & 0,6 & 1,30 & 40,30 \\
\hline Ene 02 -Ene 08 & 19:00:00 & 27,5 & 29,1 & 0,20 & 61,60 & 1,2 & 0,6 & 1,10 & 30,50 \\
\hline Ene 02 -Ene 08 & 20:00:00 & 27,4 & 29,0 & 0,10 & 63,64 & 1,2 & 0,6 & 1,30 & 40,30 \\
\hline Ene 02 -Ene 08 & 21:00:00 & 26,9 & 28,3 & 0,10 & 64,80 & 1,2 & 0,6 & 1,10 & 30,50 \\
\hline Ene 02 -Ene 08 & 22:00:00 & 26,2 & 27,8 & 0,10 & 66,90 & 0,8 & 1,2 & 0,70 & 15,30 \\
\hline Ene 02 -Ene 08 & 23:00:00 & 25,0 & 27,7 & 0,10 & 70,30 & 0,8 & 1,2 & 0,50 & 10,20 \\
\hline
\end{tabular}

(2019).

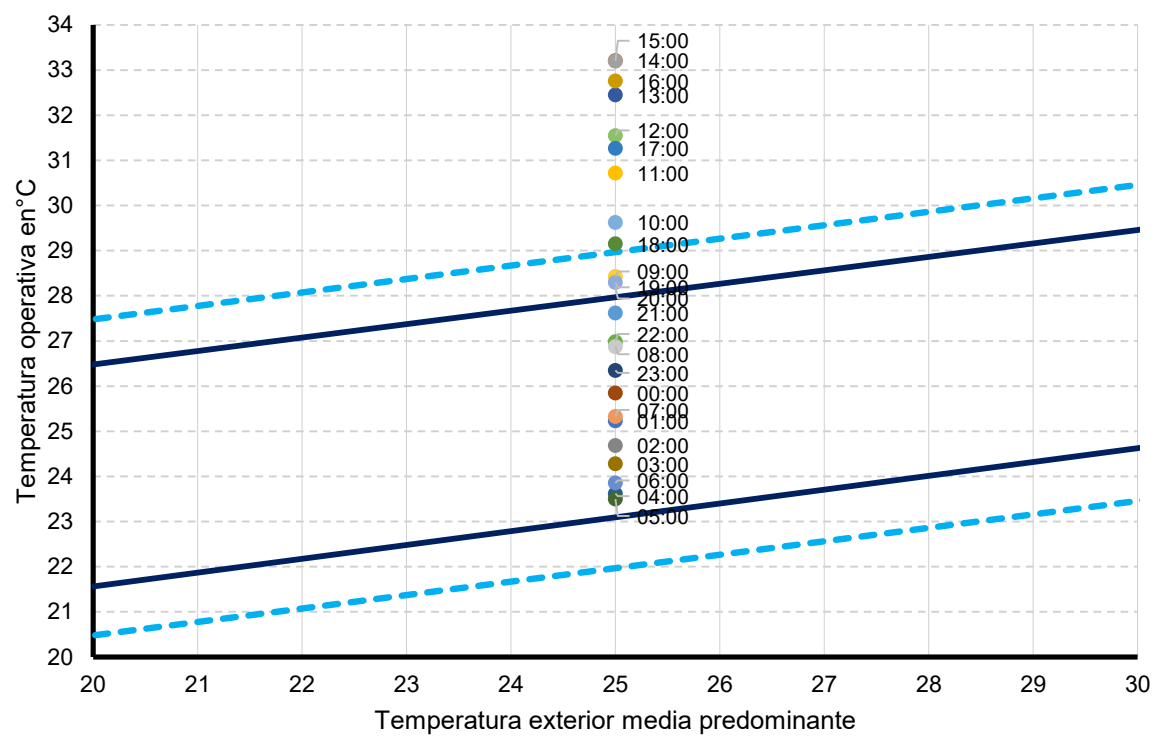

Con los resultados mostrados en las figuras 8,9 y 10 , se concibe que el nivel de aislamiento que tienen estas viviendas en sus envolventes verticales opacas es insuficiente para proveer confort térmico a sus ocupantes. Esto se refuerza con los registros de la $T_{\mathrm{mr}}$ que sobrepasan los límites máximos del rango de confort; incluso, llegan a presentarse valores medidos de hasta $48{ }^{\circ} \mathrm{C}$ en la temperatura superficial interna de la fachada oeste.

Por último, otro aspecto transversal a las VIS que se halló en el análisis de la $T_{\mathrm{mr}}$ y que también involucra la protección solar, son las cortas dimensiones de los espacios, que no dan flexibilidad a distribuciones más adecuadas del mobiliario de la alcoba, lo cual hace obligatorio que el cuerpo de los ocupantes quede próximo a las fachadas con mayor temperatura superficial.

Por otro lado, en cuanto a la ventilación natural se encontró que tan solo el $30 \%$ de la envolvente está constituida por ventanas, cuando 
lo recomendado es el $40 \%$, no solo para ventilar, sino también, para efectos de iluminación natural (Ministerio de Vivienda, Ciudad y Territorio, 2015). La baja relación ventana-pared se agrava, porque las ventanas son corredizas y solo permiten aprovechar el $50 \%$ del vano para ventilar, y bien podrían ser reemplazadas por otras de tipo batiente o en celosía, con las cuales se aprovecharía el $100 \%$ y tienen un costo levemente menor. Además, el diseño de las puertas es completamente cerrado, y también podría mejorarse al incluir aberturas superiores e inferiores, para propiciar la ventilación cruzada sin interferir con la privacidad.

Estas características de las VIS multifamiliares que ocasionan las bajas prestaciones de confort térmico son comunes a las mencionadas en los estudios para VIS unifamiliares, según lo expresado en los estudios de Gamboa et al. (2011), Iturre (2013) y Barona (2016); sin embargo, se halla una nueva: la proximidad de los cuerpos de los ocupantes a la envolvente que tiene alta temperatura superficial, situación dada por la disposición del mobiliario planteada en el diseño arquitectónico y por las pequeñas dimensiones en planta del espacio.

En lo que concierne al tema de los modelos evaluativos de confort, se encontró que utilizando el modelo PMV-PPD la sensación térmica del ambiente desde las 13:00 hasta las 17:00 alcanza lo "muy caluroso", con lo cual los porcentajes de insatisfechos llegan hasta el 93,4\%; y durante 14 de las 24 horas del día la aceptación es menor que el $80 \%$. Por su parte, mediante el modelo adaptativo también se encontraron periodos de insatisfacción, pero solo durante 9 de las 24 horas del día. Con lo expuesto, y considerando que lo revisado en el estado del arte señala al modelo adaptativo como el más preciso para evaluar el confort térmico en recintos ventilados naturalmente (Carlucci et al., 2018; Forgiarini Rup et al., 2015), se entiende que usarlo desde las etapas proyectuales significa un ahorro en materiales de aislamiento en la etapa constructiva. También, en la etapa de funcionamiento implica ahorros energéticos y económicos, debido a que el periodo en el cual se programará la climatización mecánica será en el $35 \%$ menor. Como se dijo en la primera parte del presente artículo, este tipo de ahorros en materiales y energía es necesario para disminuir la emisión de GEI generados en la producción de materiales de construcción y de electricidad, así como para evitar la construcción de nuevas hidroeléctricas (International Energy Agency, s.f.; Giraldo et al., 2015; Revista Dinero, 2019).

Como último punto, cabe mencionar que, pese a las notorias diferencias morfológicas entre tipologías VIS, los resultados obtenidos en el presente estudio al evaluar un espacio de tipología multifamiliar (PMV máximo de 2,5 o muy caluroso y PPD máximo del $93,4 \%)$ son levemente menos insatisfactorios, pero muy similares, a los encontrados por Gamboa et al. (2011), Iturre (2013) y Barona (2016) en tipología unifamiliar, quienes identificaron valores de PMV de entre 2,6 y 3,0 "muy calurosos" y PPD del $95,3 \%$ al $100 \%$; sin embargo, esto evidencia que la insatisfacción térmica es un problema generalizado en las VIS relacionado con su diseño, su materialidad y sus sistemas constructivos, y refuerza así lo encontrado por las investigaciones anteriores acerca de lo inconfortable que resulta la permanencia en ellas durante algunos periodos del día.

\section{Conclusiones}

Esta investigación monitoreó y evaluó el confort térmico de un ambiente de VIS perteneciente a la tipología multifamiliar en Cali, Colombia, caracterizada por un clima cálido seco, mediante dos modelos: PMV-PPD y adaptativo. Teniendo en cuenta que el estado del arte señala al modelo adaptativo como el más preciso para valorar edificaciones ventiladas naturalmente, se encontró que el modelo PMV-PPD exige aislamientos térmicos mayores y un consumo de energía dedicada a climatización mayor en el $35 \%$ que el modelo adaptativo, lo cual debe considerarse en términos de ahorro de recursos y energía para frenar el cambio climático. Esta consideración debe trasladarse también al Reglamento 0549 de Construcción Sostenible, ya que en la actualidad utiliza como modelo el PMV-PPD

Se concluye que la causa principal de los altos niveles de insatisfacción térmica brindados por las VIS multifamiliares a sus ocupantes no es la falta de recursos económicos, sino la falta de innovación y aplicación de los conocimientos de bioclimática.

De esta manera, el estudio esclarece que las viviendas sociales colombianas en climas cálidos requieren intervenciones que mejoren el nivel de confort térmico y garanticen la salubridad; de acuerdo con lo expuesto en la introducción, las mejores soluciones desde los puntos de vista económico y sostenible son las estrategias de enfriamiento pasivo, las cuales requieren poca o ninguna inversión adicional, tales como ventilación natural cruzada, protección solar a elementos vidriados y reducción de la absortividad y transmitancia térmica a la envolvente.

Finalmente, si en Colombia se quiere disminuir la demanda energética y aportar de verdad desde el sector de la construcción a la reducción del calentamiento global, las VIS deben ser incluidas en el cumplimiento obligatorio del Reglamento de Construcción Sostenible, y no tener cumplimiento optativo, como en el presente. Los resultados de esta investigación, sumados a los de los otros trabajos citados en el estado del arte, justifican la inclusión. En caso de que se incluyan, debe prestarse especial atención a que los ahorros energéticos se relacionen con la prestación de adecuados niveles de confort térmico, lumínico y de calidad del aire a los usuarios. Lo anterior implica intervenir desde la etapa proyectual para edificaciones nuevas y un trabajo remedial para las existentes. 


\section{Referencias}

American Society of Heating Refrigerating and Air Conditioned Engineers [ASHRAE]. (2017). ASHRAE STANDARD 55-2017 Thermal environmental conditions for human occupancy. Atlanta.

American Society of Heating Refrigerating and Air-conditioning engineers [ASHRAE]. (2001). Handbook Fundamentals. Atlanta.

Asociación Colombiana de Ingeniería Sísmica. (2010). Norma Sismoresistente 2010. Asociación Colombiana de Ingeniería Sísmica.

Barona, J. (2016). Análisis y evaluación del comportamiento térmico de dos envolventes arquitectónicas utilizadas en la vivienda de interés social en santiago de Cali. (tesis de Maestría). Santiago de Cali, Universidad del Valle.

Carlucci, S., Bai, L., de Dear, R., y Yang, L. (2018). Review of adaptive thermal comfort models in built environmental regulatory documents. Building and Environment, 137, 73-89.

https://doi.org/10.1016/j.buildenv.2018.03.053

Congreso de Colombia. (1997, 18 de julio). Ley 388 de 1997. Ley de desarrollo territorial. Diario Oficial 43.091, de 24 de julio de 1997.

http://www.secretariasenado.gov.co/senado/ basedoc/ley_0388_1997.html

Departamento Nacional de Estadística (DANE). (Febrero de 2017). DANE. http://www. dane.gov.co

Department of Technology and Built Environment, Laboratory of Ventilation and Air Quality - University of Gävle. (s.f.). PMV Calc v2. Gävle.

Dunkle, R. (1963). Configuration factors for radiant heat-transfer calculations involving people. Heat Transfer, 85(1), 71-76. https://doi.org/10.1115/1.3686012

Echeverry Campos, D., y MetroVivienda. (2000). Vivienda de interés social: Inventario de sistemas constructivos. Universidad de los Andes, Departamento de Ingeniería Civil y Ambiental, Area de Ingeniería y Gerencia de la Construcción.

Fanger, P. O. (1970). Thermal Comfort. Mc Graw Hill.

Forgiarini Rup, R., Giraldo Vásquez, N., y Lamberts, R. (2015). A review of human thermal comfort in the built environment. Energy and Buildings, 105, 178-205.

h t t p : //dx.doi.org/10.1016/j. enbuild.2015.07.047

Gamboa, J., Rosillo, M. E., Herrera Cáceres, C. A., López Bernal, O., y Iglesias, V. (2011) Confort ambiental en vivienda de interés social en Cali. Universidad del Valle.

García López, J. D., Stand Villareal, F., Gómez Cabrera, A., y Nuñez Moreno, F. A. (2016). Comparación de los principales sistemas constructivos de VIS en Colombia, desde una perspectiva de sostenibilidad, empleando BIM: caso estudio en Soacha. Pontificia Universidad Javeriana.

https://repository.javeriana.edu.co/ handle/10554/19639

Giraldo, C., Bedoya, C., y Alonso, L. (2015) Eficiencia energética y sostenibilidad en la Vivienda de Interés Social en Colombia. En Greencities \& Sostenibilidad Convocatoria de Comunicaciones Científicas (pp.155180). Universidad Politécnica de Madrid. http://oa.upm.es/42543/

Giraldo, W., y Herrera, C. (2017). Ventilación pasiva y confort térmico en vivienda de interés social en clima ecuatorial. Ingeniería y desarrollo, 35(1), 77-101. http://dx.doi.org/10.14482/inde.35.1.8944

Givoni, B. (1976). Man, climate and architecture. Applied Science Publishers.

Instituto de Hidrología, Meteorología y Estudios Ambientales, Tiempo y Clima en Colombia [IDEAM]. (16 de mayo de 2016). IDEAM. http://www.ideam.gov.co/web/tiempo-yclima/clima,2016.

International Energy Agency. (s.f.). International Energy Agency. https://www.iea.org/ statistics $/$ ?country $=$ INDIA\&year $=2016 \&$ category $=$ Energy\%20consumption\&indicator $=$ TFCShareBySector $\&$ mode $=$ chart $\&$ da taTable $=$ BALANCES

International Organization for Standardization. (2002). ISO 7726: Ergonomics of the thermal environment - Instruments for measuring physical quantities. International Organization for Standardization.

International Organization for Standardization. (2005). Ergonomía del ambiente térmico. Determinación analítica e interpretación del bienestar térmico mediante el cálculo de los índices PMV y PPD y los criterios de bienestar térmico local (ISO 7730:2005). International Organization for Standardization.

Iturre Campiño, A. (2013). Proyectar mejoras del confort térmico en la vivienda de interés social Buenaventura Caso: Barrio ciudadela Nueva Buenaventura. Cali.

Kaushik, A., Arif, M., Tumula, P., y Ebohon, O. J. (2020). Effect of thermal comfort on occupant productivity in office buildings: Response surface analysis. Building and Environment, 180, 107021. https://doi. org/10.1016/j.buildenv.2020.107021

Ministerio de Ambiente, Vivienda y Desarrollo Territorial, República de Colombia. (2011). Calidad en la vivienda de interés social (Series Guías de Asistencia Técnica para Vivienda de Interés Social). Nuevas Ediciones S.A.

Ministerio de Minas y Energía. (2009). Resolución 181331 del 6 de agosto. Reglamento Técnico de lluminación y Alumbrado Publico. https://www.minenergia. gov.co/documents/10180/23931303/ RES181331_2009.pdf/53f7e906-94de4cba-959d-eff45b04e93c6

Ministerio de Minas y Energía. (2013). Resolución 90708 del 30 de agosto. Reglamento Técnico de Instalaciones Eléctricas RETIE. https://www.minenergia.gov.co/ documents/10180/1179442/Anexo+General+del+RETIE + vigente + actualizado $+a+2015-1 . p d f / 57874 c 58-e 61 e-4104$ 8b8c-b64dbabedb13

Ministerio de Vivienda, Ciudad y Territorio. (2015). Resolución 0549 - Parámetros y lineamientos de construcción sostenible Guía para el ahorro de agua y energía en edificaciones. Reglamento del Capítulo 1 del Título 7 de la parte 2, del libro 2 del Decreto 1077 de 2015. https://camacol.co/ sites/default/files/Resoluci\%C3\%B3n\%20 549\%20del\%2010072015_0.pdf
Ministerio de Vivienda, Ciudad y Territorio (MINVIVIENDA). (2020). Reglamento Técnico del Sector de Agua Potable y Saneamiento Básico (RAS).

http://www.minvivienda.cov.co/viceministeriode-agua/reglamento-tecnico-del-sector/ reglamento-tecnico-del-sector-de-agua-potable

Olgyay, V. (1998.). Arquitectura y clima: Manual de diseño bioclimático para arquitectos y urbanistas. Gustavo Gili.

Ormandy, D., y Ezratty, V. (2012). Health and thermal comfort: From WHO guidance to housing strategies. Energy Policy. 116-121. https://doi.org/10.1016/j. enpol.2011.09.003

Planeación Municipal, Alcaldía de Santiago de Cali. (2014). Plan de Ordenamiento Territorial de Cali. http://www.cali.gov.co/planeacion/publicaciones/106497/pot_2014 idesc/

Revista Dinero. (24 de 1 de 2019). Los desafíos ambientales en Hidroituango. https://www. dinero.com/edicion-impresa/pais/articulo/el-impacto-ambiental-por-la-emergencia-de-hidroituango/266409

Santamouris, M., y Asimakopoulos, D. (2001). Passive cooling of buildings. James \& James.

Sistema Intercomunicado Nacional. (22 de Febrero de 2018). Sistema Intercomunicado Nacional. http://informesanuales.xm.com. co/2015/SitePages/operacion/2-4-Generaci\%C3\%B3n-del-SIN.aspx

United Nations Commission on Human Settlements (ONU). (2011). Informe mundial sobre asentamientos humanos: Las ciudades y el cambio climático: orientaciones para políticas. Programa de las Naciones Unidas para los asentamientos urbanos. https://www.zaragoza.es/contenidos/ medioambiente/onu/538-spa-ed2011-sum. pdf

\section{Listado de siglas}

ASHRAEAmerican Society of Heating Refrigerating and Air Conditioned Engineers.

Clo Nivel de aislamiento térmico por

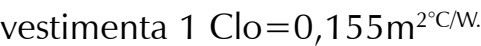

GEI Gases de efecto invernadero.

$\mathrm{Hr}$ Humedad relativa.

Met Tasa metabólica.

PMV Índice de sensación térmica (por las iniciales en inglés de Predicted Mean Vote).

PPD Índice de personas insatisfechas (por las iniciales en inglés de Predicted Percentage Dissatisfied)

$\mathrm{T}_{\text {air }}$ Temperatura del aire, o bulbo seco.

$\mathrm{T}_{\mathrm{mr}}$ Temperatura media radiante.

$\mathrm{T}_{\mathrm{op}}$ Temperatura operativa.

TRM Tasa representativa del mercado.

$V_{\text {air }}$ Velocidad del aire.

VIS Vivienda de interés social. 
A Portada: Catedral de Notre Dame de Paris durante los trabajos de reparación en octubre de 2020 Fotografía: Arquitecto Andrés Avila Gómez (octubre, 2020)
(A) Orientación editorial

\section{Enfoque y alcance}

La Revista de Arquitectura (Bogotá) ( (ISSN 1657-0308 Impresa y E-ISSN 2357-626X en línea) es una publicación científica seriada de acceso abierto, arbitrada mediante revisión por pares (doble ciego) e indexada, en donde se publican resultados de investigación originales e inéditos.

Está dirigida a la comunidad académica y profesional de las áreas afines a la disciplina. Es editada por la Facultad de Diseño y el Centro de Investigaciones (CIFAR) de la Universidad Católica de Colombia en Bogotá (Colombia).

La principal área científica a la que se adscribe la Revista de Arquitectura (Bogotá) según la OCDE es:

Gran área: 6. Humanidades

Área: 6.D. Arte

Disciplina: 6D07. Arquitectura y Urbanismo

También se publican artículos de las disciplinas como 2A02, Ingeniería arquitectónica; 5G03, Estudios urbanos (planificación y desarrollo); 6D07, Diseño.

Los objetivos de la Revista de Arquitectura (Bogotá) son:

- Promover la divulgación y difusión del conocimiento generado a nivel local, nacional e internacional

- Conformar un espacio para la construcción de comunidades académicas y la discusión en torno a las secciones definidas.

- Fomentar la diversidad institucional y geográfica de los autores que participan en la publicación.

- Potenciar la discusión de experiencias e intercambios científicos entre investigadores y profesionales.

- Contribuir a la visión integral de la arquitectura, por medio de la concurrencia y articulación de las secciones mediante la publicación de artículos de calidad.

- Publicar artículos originales e inéditos que han pasado por revisión de pares, para asegurar que se cumplen las normas éticas, de calidad, validez científica, editorial e investigativa.

- Fomentar la divulgación de las investigaciones y actividades desarrolladas en la Universidad Católica de Colombia.
Palabras clave de la Revista de Arquitectura (Bogotá): arquitectura, diseño, educación arquitectónica, proyecto y construcción, urbanismo.

Idiomas de publicación: español, inglés, portugués y francés.

Título abreviado: Rev. Arquit.

Titulo corto: RevArq

\section{Políticas de sección}

La revista se estructura en tres secciones correspondientes a las líneas de investigación activas y aprobadas por la institución, y dos complementarias, que presentan dinámicas propias de la Facultad de Diseño y las publicaciones relacionadas con la disciplina.

Cultura y espacio urbano. En esta sección se publican los artículos que se refieren a fenómenos sociales en relación con el espacio urbano, atendiendo aspectos de la historia, el patrimonio cultural y físico, y la estructura formal de las ciudades y el territorio.

Proyecto arquitectónico y urbano. En esta sección se presentan artículos sobre el concepto de proyecto entendido como elemento que define y orienta las condiciones proyectuales que devienen en los hechos arquitectónicos o urbanos, y la forma como estos se convierten en un proceso de investigación y nuevo de conocimiento. También se presentan proyectos que sean resultados de investigación, los cuales se validan por medio de la ejecución y transformación en obra construida del proceso investigativo. También se contempla la publicación de investigaciones relacionadas con la pedagogía y didáctica de la arquitectura, el urbanismo y el diseño.

Tecnología, medioambiente y sostenibilidad. En esta sección se presentan artículos acerca de sistemas estructurales, materiales y procesos constructivos, medioambiente y gestión, relacionados con los entornos social-cultural, ecológico y económico.

Desde la Facultad. En esta sección se publican artículos generados en la Facultad de Diseño, relacionados con las actividades de docencia, extensión, formación en investigación o internacionalización, las cuales son reflejo de la dinámica y de las actividades realizadas por docentes, estudiantes y egresados; esta sección no puede superar el $20 \%$ del contenido.

Textos. En esta sección se publican reseñas, traducciones y memorias de eventos relacionados con las publicaciones en Arquitectura y Urbanismo.
A Frecuencia de publicación

Desde 1999 y hasta el 2015, la Revista de Arquitectura (Bogotá) publicó un volumen al año, a partir del 2016 se publicarán dos números por año en periodo anticipado, enero-junio y julio-diciembre, pero también maneja la publicación anticipada en línea de los artículos aceptados (versión Post-print del autor).

La Revista de Arquitectura (Bogotá) se divulga mediante versiones digitales (PDF, HTML, EPUB, XML) e impresascon un tiraje de 700 ejemplares, los tiempos de producción de estas versiones dependerán de los cronogramas establecidos por la editorial.

Los tiempos de recepción-revisión-aceptación pueden tardar entre seis y doce meses dependiendo del flujo editorial de cada sección y del proceso de revisión y edición adelantado.

Con el usuario y contraseña asignados, los autores pueden ingresar a la plataforma de gestión editorial y verificar el estado de revisión, edición o publicación del artículo.
(A) Canje

La Revista de Arquitectura (Bogotá) está interesada en establecer canje con publicaciones académicas, profesionales o científicas del área de Arquitectura y Urbanismo, como medio de reconocimiento y discusión de la producción científica en el campo de acción de la publicación.

Mecanismo

Para establecer canje por favor descargar, diligenciar y enviar el formato: RevArq FP20 Canjes

Universidad Católica de Colombia
(202I, enero-junio). Revista de
Arquitectura (Bogotá), 23(I),
I-I24. Doi: 10.14718

ISSN: I657-0308
E-ISSN: 2357-626X
Especificaciones:
Formato: $34 \times 24 \mathrm{~cm}$
Papel: Mate II5 g
Tintas: Negro y policromía

Contacto

Dirección postal:

Avenida Caracas No. 46-72

Universidad Católica de Colombia

Bogotá D. C., Colombia

Código postal: 111311

Facultad de Diseño

Centro de Investigaciones (CIFAR)

Sede El Claustro. Bloque "L", 4 piso

Diag. 46A No. 15b-10

Editor, Arq. César Eligio-Triana

Teléfonos:

+57 (1) $3277300-3277333$

Ext. 3109; 3112 o 5146

Fax: +57 (1) 2858895
Correo electrónico:

revistadearquitectura@ucatolica.edu.co cifar@ucatolica.edu.co

Página WEB:

www.ucatolica.edu.co

Vínculo Revistas científicas

http://publicaciones.ucatolica.edu.co revistas-cientificas

https://revistadearquitectura.ucatolica.edu.co/ 
Reflexiones en torno a la enseñanza de la arquitectura y el urbanismo en Colombia. Conversaciones con Juan Carlos

m. Pérgolis Valsecchi, René Carrasco Rey y Juan Carlos del

¿ Castillo

Reflections on the teaching of architecture and urban planning in Colombia. Conversations with Juan Carlos Pérgolis Valsecchi, René Carrasco Rey y Juan Carlos del Castillo

웅

La experiencia de caminar en ciudades latinoamericanas

The experience of walking in Latin American cities

Movilidad urbana de la población en la ciudad de Encarnación * Paraguay. Desarrollo urbano y gestión ambiental

$m$ Urban mobility of the population in the city of Encarnación, Paraguay.

这

ษั

La vivienda adecuada financiada según el ingreso.

El discurso de las Naciones Unidas

பं

La arquitectura frente a las innovaciones pedagógicas.

Pervivencia y resignificación de la Escuela Nueva en el Cono Sur Architecture facing the pedagogical innovations. Survival and resignification of the New School in the Southern Cone

Aporte de las competencias investigativas a la integración de saberes curriculares.

Una visión en el escenario de aprendizaje del diseño

arquitectónico

Contribution of research competencies to the integration of curricula knowledge. A vision in the architectural design learning scenario

Adobe como saber ancestral usado en construcciones

N autóctonas de Pore y Nunchía, Casanare (Colombia)

j. Adobe as an ancestral knowledge used in autochthonous constructions $\stackrel{4}{\longleftarrow}$

Paisaje construido y sustentabilidad urbana: huellas

$œ$

El Plan de Transformación de Osorno

Built landscape and urban sustainability: Identity prints of the modern landscape. The Osorno Transformation Plan

Indicadores de sostenibilidad social y su relación con el

$\hat{a}$

ن்

노 caso de estudio en Cuenca (Ecuador)

ن

Confort térmico en vivienda social multifamiliar de clima cálido en Colombia

느 Thermal comfort in multi-family social housing in a warm climate in ن் Colombia
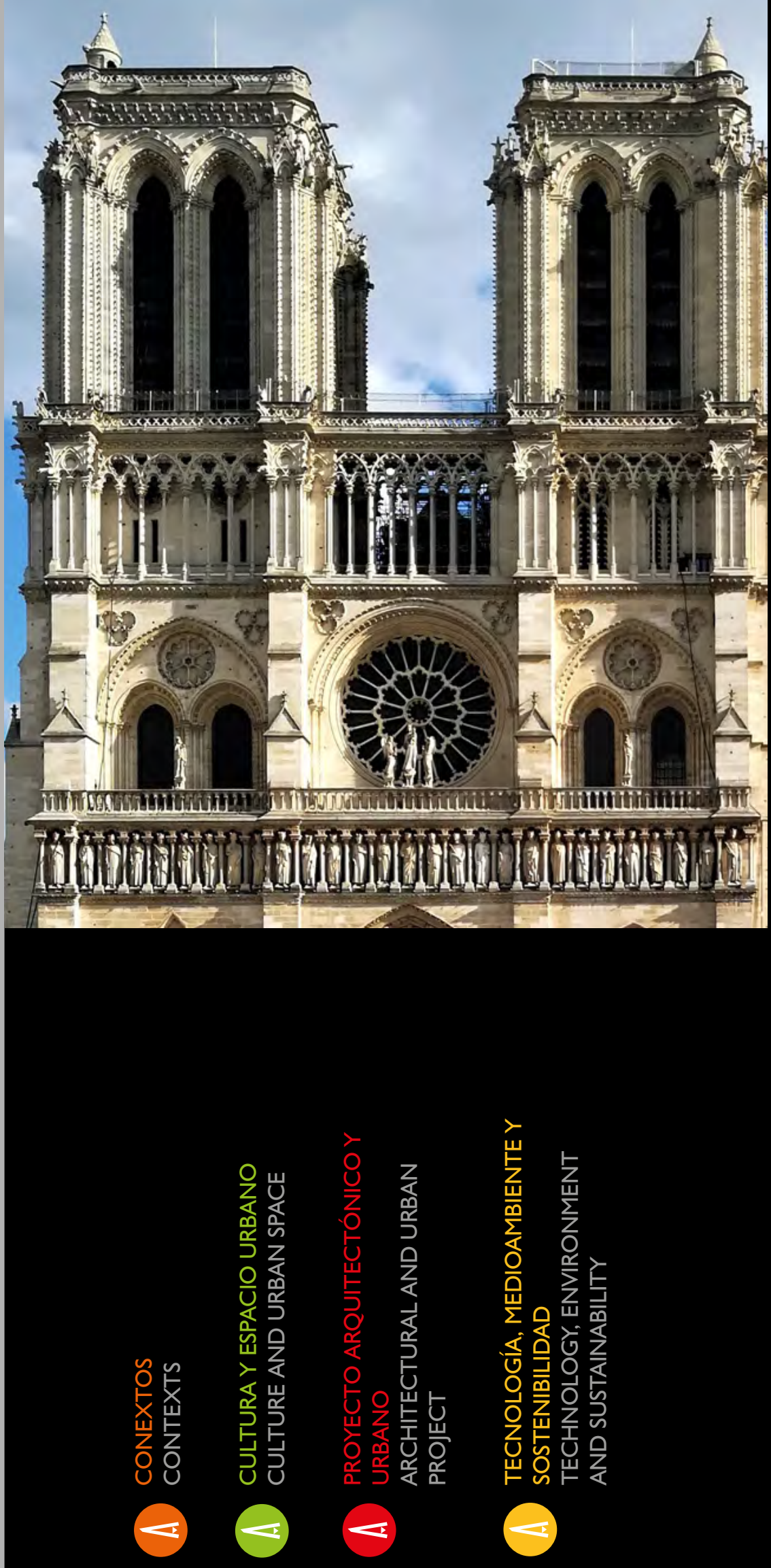

La Revista de Arquitectura es de acceso abierto, arbitrada e indexada y está presente en:
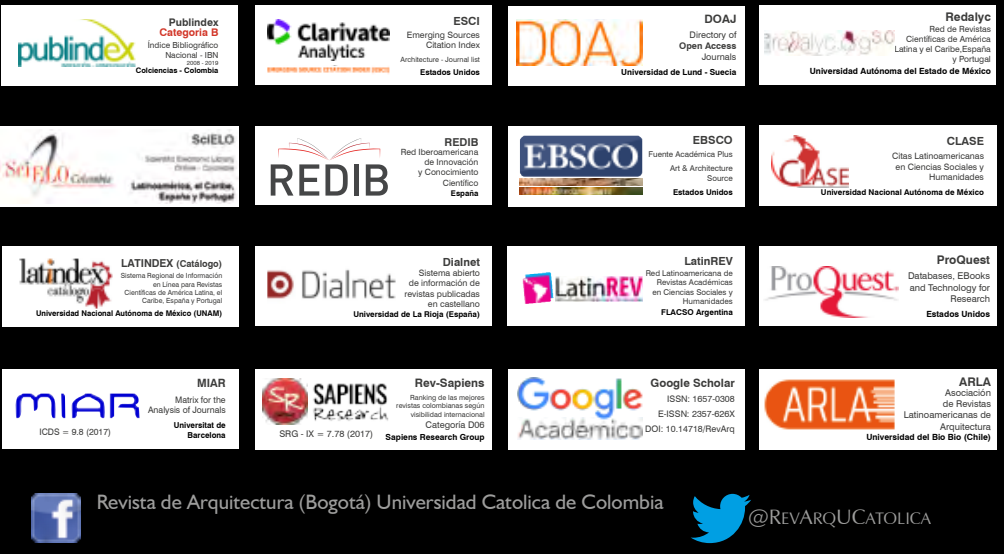

8
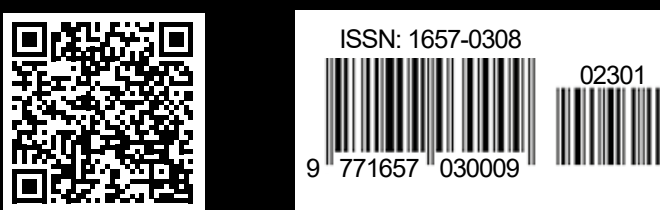